\title{
TAMAÑO DE PUESTA, SITIOS DE NIDIFICACIÓN Y ÉXITO REPRODUCTIVO DEL CAIQUÉN (CHLOEPHAGA PICTA GMELIN, 1789) EN LA RESERVA DE BIOSFERA CABO DE HORNOS, CHILE
}

\author{
CLUTCH SIZE, NESTING SITES, AND BREEDING SUCCESS OF THE UPLAND GOOSE \\ (CHLOEPHAGA PICTA GMELIN, 1789) IN THE CAPE HORN BIOSPHERE RESERVE, CHILE
}

\author{
José Tomás Ibarra ${ }^{1,2}$, Elke Schüttler ${ }^{1,3,4}$, Steven McGehee ${ }^{1,5}$ \& Ricardo Rozzi ${ }^{1,6}$ \\ RESUMEN
}

Los estudios sobre la biología del caiquén (Chloephaga picta) en los ecosistemas subantárticos de Chile son escasos y, fundamentalmente, constituyen registros anecdóticos sobre la especie. Con el fin de aportar en su conocimiento, se estudió su biología reproductiva en términos del tamaño de puesta, sitios de nidificación y éxito reproductivo en función de variables del hábitat en isla Navarino (54-55 $5^{\circ}$ ), Reserva de Biosfera Cabo de Hornos (RBCH), Chile. Se monitorearon 79 nidos en hábitats costeros durante 54 días del período reproductivo en 2005/2006 y durante 59 días en 2006/2007. El tamaño de puesta fue 6,1 $\pm 1,2$ huevos. Los nidos fueron preferentemente construidos en aquellos sitios costeros con pendiente baja $\left(0-10^{\circ}\right)$, en hábitat de matorral $(70,5 \%)$, dominado por Chiliotrichum diffussum $(30,8 \%)$ y Berberis buxifolia (24,4\%). Las piedras fueron el elemento mayoritario en los hábitats de nidificación (50,1\%). La cobertura vegetacional sobre el nido fue baja (0-25\%) en un 82,1\% de los nidos, y la adyacente fue intermedia (25-50\%) en un $29,5 \%$ de ellos. Un $39,7 \%$ de los nidos fue construido en hábitats con una cobertura relativamente alta (>40\%). En total, un 36,7\% de los nidos presentaron eclosión de huevos (exitosos según método tradicional) y un $57,0 \%$ de los nidos fueron depredados. No se encontró una asociación del éxito o fracaso de nidificación a variables de hábitat. La probabilidad de éxito reproductivo fue de un 20,0\% (método Mayfield). Ambos valores calculados están por debajo de los reportados para otras especies de gansos (>60\%) y, también, de los reportados para esta especie $(61-75 \%)$ en islas Malvinas-Falklands ( $<300 \mathrm{~km}$ de isla Navarino). Este estudio muestra la necesidad de conocer más aspectos de la biología y conservación de $\mathrm{C}$. picta en la $\mathrm{RBCH}$, particularmente cuando se consideran los cambios ambientales (especies invasoras, desarrollo turístico, cambio climático, etc.) que están ocurriendo en los sistemas subantárticos.

Palabras clave: biología reproductiva, Chloephaga picta, depredación, éxito reproductivo, isla Navarino, Mayfield, tamaño de puesta, sub-antártico.

\footnotetext{
ABSTRACT

Studies on the biology of the Upland Goose (Chloephaga picta) in Chilean Sub-Antarctic ecosystems are scarce and basically constitute anecdotic records of the species. With the aim to broaden this knowledge, we studied its breeding biology in terms of clutch size, nesting sites and breeding success as a

1Parque Etnobotánico Omora (Sede Puerto Williams Universidad de Magallanes, Instituto Milenio de Ecología y Biodiversidad, Fundación Omora), Puerto Williams, Chile. jtibarra@uc.cl

${ }^{2}$ Laboratorio Fauna Australis, Programa de Recursos Naturales, Facultad de Agronomía e Ingeniería Forestal, Pontificia Universidad Católica de Chile. Vicuña Mackenna 4860, P.O. Box 306-22, Macul, Santiago, Chile

${ }^{3}$ Department of Conservation Biology. Helmholtz Centre for Environmental Research-UFZ, Permoserstr. 15, 04318 Leipzig, Alemania

${ }^{4}$ Lehrstuhl für Landschaftsökologie, Technische Universität München-Weihenstephan, Am Hochanger 6, 85350 Freising, Alemania

${ }^{5}$ Department of Biology, University of Victoria, Victoria, B.C. Canada V8W 3N5

${ }^{6}$ Department of Philosophy \& Religion Studies, University of North Texas, Denton, TX 76201, EE.UU.
} 
function of habitat variables on Navarino Island (54$\left.55^{\circ} \mathrm{S}\right)$, Cape Horn Biosphere Reserve (CHBR), Chile. We monitored 79 nests in coastal habitats during 54 days of breeding activity in the nesting season of 2005/2006 and 59 days in 2006/2007. Clutch size was $6.1 \pm 1.2$ eggs. Nests were preferably built on coastal sites with low slope $\left(0-10^{\circ}\right)$. The dominant breeding habitat was shrubland $(70.5 \%)$ dominated by Chiliotrichum diffussum (30.8\%) and Berberis buxifolia (24.4\%). Pebbles were the most common element on the nesting sites (50.1\%). The overhead nest concealment was low $(0-25 \%)$ in $82.1 \%$ of the nests, and lateral coverage was intermediate (25-50\%) in $29.5 \%$. $39.7 \%$ of the nests were built in habitats with relatively high vegetation cover (>40\%). In total, $36.7 \%$ of the clutches hatched (successful following the traditional method) and 57,0\% were predated. Measured habitat variables did not explain success or failure of the nests. The nest survival probability was 20,0\% (Mayfield method). The two calculated values are lower than those reported in other geese species $(>60 \%)$ and also than values $(61-75 \%)$ reported for this species in Falkland-Malvinas Islands $(<300 \mathrm{~km}$ from Navarino Island). This study shows the need of understanding more aspects of the biology and conservation of $\mathrm{C}$. picta in the $\mathrm{CHBR}$, in particular when considering the environmental changes (invasive species, tourism development, climate change, etc) currently going on in the Sub-Antarctic systems.

Key words: breeding biology, Chloephaga picta, clutch size, Mayfield, Navarino Island, nesting success, predation, Sub-Antarctic.

\section{INTRODUCCIÓN}

El Caiquén (Chloephaga picta Gmelin, 1789) constituye una de las cinco especies de gansos silvestres del género Chloephaga presentes en Chile, y es endémica del neotrópico (Summers \& McAdam 1993). La subespecie C. p. picta se distribuye desde la cordillera de Santiago hasta el archipiélago cabo de Hornos (Martínez \& González 2004), constituyendo la especie más abundante de ambientes húmedos de la Patagonia chilena (Garay et al. 1991). También es una de las aves más numerosas en la isla grande de Tierra del Fuego (Humphrey et al. 1970, Earnshaw 1973, Schlatter et al. 2002), y en las islas al sur del canal Beagle (Barros 1971, Couve \& Vidal 2003). Parte de su población ha sido descrita nidificando desde la zona central de Chile y Argentina al sur
(Couve \& Vidal 2003), aunque su reproducción ha sido solamente estudiada de manera descriptiva en Tierra del Fuego (Jory et al. 1974). Estudios cuantitativos se han desarrollados en zonas meridionales como las islas Malvinas, sobre la subespecie C. picta leucoptera (e.g. Harradine 1977, Summers 1983, Summers \& McAdam 1993, Quillfeldt et al. 2005). Estos últimos estudios han sido originados, fundamentalmente, debido al conflicto existente entre la actividad ganadera y esta ave, la cual constituiría un competidor de la actividad, debido a sus hábitos alimenticios herbívoros (Summers \& McAdam 1993).

Para el caso del extremo sur de Chile, específicamente la isla Navarino, los estudios desarrollados en la especie, hasta ahora, señalan: algunos de sus patrones migratorios (Ibarra 2007) 1 y estimación de sus densidades a lo largo de la costa norte y vulnerabilidad a la depredación por el visón americano Neovison vison (Schüttler et al. 2009). Sin embargo, su biología reproductiva aún no ha sido estudiada en la Patagonia chilena. En este contexto, surge la necesidad de ampliar el conocimiento básico sobre esta especie en la Reserva de Biosfera Cabo de Hornos (RBCH), donde C. picta es la especie más abundante en los ambientes húmedos del área (Ibarra et al. 2009a).

La depredación es el factor dominante que afecta el éxito reproductivo de la avifauna y puede tener un rol fundamental en la dinámica poblacional de las aves (Wilcove 1985, Klett et al. 1988, Newton 1998). Como consecuencia, la selección debería favorecer a aquellas aves que usan estrategias o que seleccionan sitios que minimizan el riesgo de depredación. Una serie de factores pueden afectar la vulnerabilidad del nido a la depredación, pero uno de los más importantes lo constituye el grado de ocultamiento del nido. La selección de hábitats que presenten una mayor complejidad, microrelieves y cobertura vegetacional, deberían reducir la visibilidad del nido y, por lo tanto, el riesgo de depredación en las especies que nidifican a nivel de suelo (e.g. Hill 1984, Jackson et al. 1988, Schieck \& Hannon 1993). Sin embargo, Götmark (1992) señala que una mayor cobertura del nido podría obstruir la visión del individuo que incuba hacia sus alrededores y podría limitar una eficaz respuesta a un depredador. Esto es particularmente importante en islas oceánicas donde las especies han evolucionado libre de depredadores carnívoros, lo que determina

${ }^{1}$ Ibarra, J. T. 2007. El visón en Upushwaia: variaciones estacionales de la avifauna en ambientes de humedal y de su depredación por la especie exótica Mustela vison (Schreber) en isla Navarino, Reserva de Biosfera Cabo de Hornos, Chile. Tesis Magíster (MSc), Recursos Naturales, Facultad de Agronomía e Ingeniería Forestal, Pontificia Universidad Católica de Chile. 117 pp. 
que gran parte de ellas nidifiquen en el suelo sin mecanismos conductuales de defensa (Amarasekare 1993). Esto puede ser el caso de varias aves en isla Navarino, donde $N$. vison representa una nueva especie funcional de depredador terrestre (Rozzi \& Sherriffs 2003, Schüttler et al. 2009), junto a otras invasoras como perros bagüales (Rozzi et al. 2006).

Con el fin de aportar en el conocimiento de la biología reproductiva de C. picta picta, se desarrolló un estudio que tuvo como objetivos: (a) determinar tamaño de puesta y éxito de nidada, (b) estudiar los atributos del hábitat que esta especie selecciona para nidificar, (c) evaluar si los hábitats con mayor complejidad, micro-relieves y cobertura vegetacional, reducen el riesgo de depredación de nidos de esta especie en isla Navarino, y (d) discutir los antecedentes estudiados, incluyendo su éxito reproductivo, con el potencial efecto que podría estar teniendo N. vison sobre el éxito reproductivo de C. picta, en la $\mathrm{RBCH}$.

\section{MATERIAL Y MÉTODOS}

\section{Área de estudio}

El estudio se realizó en isla Navarino $\left(54^{\circ} 55^{\prime} \mathrm{S}\right.$ $\left.67^{\circ} 39^{\prime} \mathrm{W}\right)$, la cual es parte de la $\mathrm{RBCH}$, en el extremo austral de Chile (Fig. 1). La ecorregión corresponde a la de los bosques magallánicos subantárticos, donde los ecosistemas terrestres forman un mosaico de diversos tipos de hábitat, entre los cuales se encuentran: 1. Bosques siempreverdes, dominados por coihue de Magallanes (Nothofagus betuloides) y canelo (Drimys winteri); 2. Bosques deciduos, dominados por lenga ( $N$. pumilio) y/o ñirre (N. antarctica); 3. Hábitats de altura con formaciones de plantas en cojín y praderas de líquenes; 4. Formaciones de tundra, tales como humedales de juncáceas y turberas de Sphagnum spp.; y 5. Ecosistemas dulceacuícolas (Pisano 1977, Rozzi et al. 2006). La temperatura media anual es de $6^{\circ} \mathrm{C}$ y la precipitación media alcanza los $467,3 \mathrm{~mm}$, cayendo en forma de nieve en los meses fríos (di Castri \& Hajek 1976). La isla Navarino tiene un área aproximada de $2.528 \mathrm{~km}^{2}$, donde Puerto Williams constituye el asentamiento humano más importante del área con 2.200 habitantes (Rozzi et al. 2006).

Monitoreo de nidos y tamaño de puesta

C. picta usualmente nidifica cerca del agua en los valles, bordeando humedales y ríos, y a lo largo de la costa marina (Summers 1983). En el presente estudio, la actividad reproductiva de $C$. picta fue monitoreada en nueve sitios a lo largo de la costa norte de la isla Navarino durante 54 días del período reproductivo 2005/06, comenzando el 11 de noviembre, y 59 días de período reproductivo 2006/07, comenzando el 6 de noviembre. Se monitorearon 35 nidos en 2005/06 y 44 nidos en 2006/07 ( $n=79)$. La búsqueda de nidos se realizó utilizando siete (2005/06) y nueve (2006/07) transectos de $4 \mathrm{~km}$ de longitud a lo largo de la costa marina, en cada uno de los sitios estudiados. Con el objetivo de detectar a las aves en actividad reproductiva se recorrieron repetitivamente los transectos y se registró el comportamiento reproductivo o la presencia de un macho en actitud territorial hasta los $300 \mathrm{~m}$ desde el borde de la costa. Frecuentemente, ocho días (8,1 d, DE=1,78 d) ocurrieron entre visitas realizadas a un mismo nido. Con el fin de minimizar los efectos positivos o negativos del observador sobre la sobrevivencia de los huevos por los efectos antrópicos tales como huellas, concurrencia al nido o al comportamiento (Götmark 1992); se seleccionó una frecuencia moderada de visitas a los nidos y se redujo al mínimo el tiempo de visita en cada nido. Los nidos fueron posicionados en un mapa a $\pm 25 \mathrm{~m}$, utilizando un receptor de Sistema de Posicionamiento Global (Fig. 1). Fueron instaladas cintas de color a una distancia de $5 \mathrm{~m}$ desde el nido. El tamaño de la puesta para $C$. picta fue determinado solamente para aquellos nidos que fueron visitados al menos en dos ocasiones durante el período de incubación (Godfray et al. 1991). Los nidos fueron visitados durante: (a) el período de puesta, (b) el período de incubación, (c) en la eclosión, y (d) posterior a ésta (Fig. 2). En cada visita se registró el número de huevos hasta que se produjera la eclosión, abandono o que éstos hayan sido depredados totalmente.

\section{Sitios de nidificación}

Se caracterizaron los siguientes atributos en el hábitat de nidificación: 1) tipo de hábitat clasificado como desnudo (sin vegetación), uniforme (pastizal o turbera), mixto (arbustos y pastizal, pero sin árboles), complejo (bosque deciduo, mixto o siempreverde); 2) especie vegetal dominante en una superficie de $100 \mathrm{~m}^{2}$ alrededor del nido; 3) cobertura vegetacional 
(0,1-1 $\mathrm{m}$ de altura) en un radio de $5 \mathrm{~m}$ desde el nido (en categorías $<20 \%, 21-40 \%,>40 \%$ ); 4 ) altura (m) de arbustos adyacentes al nido cuando los había; 5) distancia $(\mathrm{m})$ directa al agua; 6) pendiente del borde costero $\left(0-10^{\circ}, 11-20^{\circ}, 21-30^{\circ},>31^{\circ}\right)$; 7) proporción (\%) de elementos en un área de $20 \mathrm{~m}^{2}$ del borde de costa (barranco, rocas $>10 \mathrm{~cm}$ de diámetro, piedras $<10 \mathrm{~cm}$, arena, barro, vegetación); 8) cobertura sobre el nido observando desde $50 \mathrm{~cm}$ por sobre éste (0-25, 25-50, 50-75, 75-100\%); y 9) cobertura adyacente al nido desde sus cuatro costados (0-25, 25-50, 50-75, 75-100\%) desde $5 \mathrm{~m}$.

\section{Éxito reproductivo y análisis de datos}

El éxito reproductivo se refiere a la proporción de aquellos nidos en que, al menos, un huevo eclosionó (Jehle et al. 2004). Para estimar el éxito reproductivo se utilizó el método tradicional (número de nidos exitosos/número de nidos totales) y el método propuesto por Mayfield (Mayfield 1961, 1975), con la estimación de la varianza desarrollada por Johnson (1979). Se calcularon dos estimaciones de Mayfield. Primero considerando un período de puesta de huevos e incubación de 36 días (Summers 1983) suponiendo una sobrevivencia de nidos constante (Jehle et al. 2004). Luego se presentaron las estimaciones estratificadas como ha sido recomendado por Jehle et al. (2004), considerando seis días de puesta de huevos y 30 días de incubación (Summers 1983). Se utilizaron regresiones logísticas con procedimiento Stepwise Mixed, para evaluar, de forma simultánea, el efecto de las variables y sus interacciones sobre el éxito de nidada. Estos análisis trataron el éxito reproductivo como una variable binaria ( $1=$ éxito, $0=$ fracaso) (Tremblay et al. 1997). Los análisis estadísticos fueron realizados con el programa SPSS versión 10.0 (SPSS 1999).

\section{RESULTADOS}

Los nidos de C. picta fueron construidos sobre el suelo y consistieron en una base de ramillas de especies arbustivas (Chilliotrichum diffussum, Berberis buxifolia, etc.), junto con pastos de la familia Poaceae. Las dimensiones de los nidos ( $\mathrm{N}=$ 24 descritos) fueron de 15,2 $\pm 3,4 \mathrm{~cm}$ de espesor y $50 \mathrm{~cm}$ de diámetro máximo. En su interior cada nido contaba de una gruesa capa de plumón, algunas plumas de cobertura y semiplumas que abrigaban los huevos (Fig. 2). En todos los casos, la hembra fue la encargada de la incubación (Fig. 2), mientras el macho generalmente permaneció en el territorio, defendiéndolo de otros machos de la misma especie.

Se visitó un total de 39 nidos de C. picta en más de una ocasión, durante el período de incubación de éstos. A partir de ellos, se determinó el tamaño de puesta, el cual fue de 6,1 \pm 1,2 huevos. Los tamaños de puesta observados en éste y otros estudios previos, realizados en distintas zonas del sur de Sudamérica, se presentan en la tabla 1.

El tipo de hábitat dominante en que nidificó C. picta fue el de matorral o mixto (70,5\%), seguido del pastizal-turbera o uniforme $(15,4 \%)$ (Fig. 3). La especie vegetal que predominó en el sitio de nidificación fue la mata negra (Chilliotrichium diffussum) $(30,8 \%)$, seguida por calafate (Berberis buxifolia) $(24,4 \%)$, especies no identificadas de la familia Poaceae $(21,8 \%)$ y chaura (Pernettya mucronata) (17,9\%). La cobertura vegetacional del hábitat en un radio de $5 \mathrm{~m}$ desde el nido, fue mayor a un $40 \%$ en un $39,7 \%$ de los nidos, seguida de una cobertura entre $20-40 \%$ en un $34,6 \%$ de ellos. Por otra parte, la altura media de los arbustos vecinos a los nidos fue de 0,24 $\pm 0,13 \mathrm{~m}$ (rango de 0 a 0,5 m). En cuanto a los sitios de nidificación, se observó que la distancia media al agua fue de 35,6 \pm 47,3 m (rango de 1 a $236 \mathrm{~m}$ ). Los nidos fueron instalados, principalmente, en sitios de pendiente baja entre $0-10^{\circ}(62 \%)$ de desnivel, seguido de pendientes entre $21-30^{\circ} \mathrm{m}(21 \%)$. Los sitios con pendientes mayores a $31^{\circ}$ fueron escasamente utilizados (4\%). Respecto a la proporción (\%) de elementos que predominan en los sitios de nidificación, se observó que las piedras fueron el elemento predominante $(50,1 \%)$, seguido de rocas $(31,2 \%)$ y barrancos $(14,9 \%)$. La cobertura de la vegetación sobre el nido fue considerablemente baja, alcanzando en un $82,1 \%$ de los casos entre $0-25 \%$, seguida de una cobertura entre $25-50 \%$ (12,8\%). La cobertura adyacente a los nidos fue variable, siendo en un $29,5 \%$ de los casos entre $25-50 \%$, seguida en un $26,9 \%$ por coberturas entre $0-25$ y $25-50 \%$.

En cuanto al estado en que se encontraron los nidos en su última visita, en un $57,0 \%$ de los casos éstos habían sido depredados. Un 36,7\% de los nidos presentaron eclosión de huevos (considerados como exitosos según el método tradicional), y un 6,3\% de ellos fueron abandonados (Fig. 4). El éxito reproductivo, o sobrevivencia de nidos, calculado utilizando el método Mayfield $(1961,1975)$ fue de un 
$20,0 \%$ (95\% CI $=12,62-31,41 \%)$ desde la puesta del primer huevo. Considerando solamente el período de puesta de huevos (seis días), la sobrevivencia de nidos fue de un 52,63\% (95\% CI = 36,96-77,36\%) $y$, considerando el período de incubación, el éxito reproductivo estimado fue de un $33,46 \%$ (95\% CI = 48,09-23,17\%). Ninguno de los atributos de hábitat estudiados se relacionó significativamente con el éxito o fracaso de las nidadas de C. picta ( $p>0,05)$. En uno de los nidos ya visitado una semana antes, se produjo un encuentro accidental con un individuo de $N$. vison justo cuando este se disponía a atacar el nido.

\section{DISCUSIÓN}

Este trabajo aborda aspectos fundamentales de la biología reproductiva de C. picta en la Reserva de Biosfera Cabo de Hornos. Los tamaños de puesta de C. picta en isla Navarino fueron similares a los reportados en estudios desarrollados en otros sistemas similares como las islas Malvinas (e.g., Harradine 1977, Summers 1983, Quillfeldt et al. 2005) y Tierra del Fuego (Jory et al. 1974) (Tabla 1). Sin embargo, los datos sugieren que el éxito de nidada de C. picta en isla Navarino es relativamente bajo y la principal causa del fracaso reproductivo lo constituiría la depredación de los nidos. El éxito reproductivo de otros anátidos (patos y gansos) normalmente supera el $60 \%$ para la mantención de poblaciones viables (e.g., Mickelson 1975, Cooper 1978, Ely \& Raveling 1984, Lepage 1998, Guyn \& Clark 2000). Para el caso de C. picta en islas Malvinas, Quillfeldt et al. (2005) encontraron un $75 \%$ de éxito reproductivo (según método tradicional), valor que es muy superior al 36,7\% encontrado en el presente estudio en la isla Navarino. Por su parte, Summers (1983) encontró valores de éxito reproductivo por sobre un $61 \%$ en islas Malvinas.

A pesar de que este trabajo no determinó las especies que están ejerciendo presión sobre los nidos de C. picta, Schüttler et al. (2009) han identificado a los depredadores responsables en el mismo set de datos (i.e., aves $17,7 \%$, personas $13,9 \%$, visón $10,1 \%$, y perros $1,3 \%$, del total de nidos). Las aves nativas de la zona responsables de la depredación incluyeron a: Caracara plancus, Milvago chimango, Stercorarius chilensis y Larus dominicanus. Sin embargo, las otras causas de pérdida de nidos son antrópicas (incluyendo especies invasoras y cosecha por personas). En este sentido, a pesar de que $N$. vison ocupó el tercer lugar entre los depredadores, este invasor podría aumentar su papel en la depredación de nidos de $C$. picta. $N$. vison fue registrado por primera vez en isla Navarino a inicios de esta década y estaría teniendo un rápido crecimiento poblacional (Rozzi \& Sherriffs 2003). En el hemisferio norte, $N$. vison y Alopex lagopus son los principales depredadores de nidos de varias especies de gansos silvestres (Summers \& Underhill 1986, Samelius \& Lee 1998), los que han evolucionado en conjunto con estos anátidos.

En isla Navarino no existen especies nativas de carnivoros terrestres (Anderson et al. 2006). Aunque, de igual manera, C. picta presentaría estrategias de defensa sólo contra depredadores aéreos de sus nidadas (Quillfeldt et al. 2005). Para el caso de N. vison, estudios recientes de su dieta han determinado que $C$. picta es el ave más consumida por este depredador en los ambientes húmedos de la Reserva de Biosfera Cabo de Hornos (Ibarra et al. 2009b). En definitiva, el impacto de N. vison en conjunto con el impacto que podrían ejercer personas que consumen los huevos de C. picta, representan dos factores que podrían afectar a la población de este anátido en los ecosistemas subantárticos. Así, estos aspectos parecen ser prioritarios de entender y considerar en futuras iniciativas de conservación de esta especie en la $\mathrm{RBCH}$.

A nivel ecosistémico, C. picta es considerada una especie crítica en el crecimiento y mantención de Tayloria dubyi, especie de musgo endémico de los bosques templados de Sudamérica (Jofré 2009)2. Este musgo se caracteriza por atraer a dípteros que se posan sobre sus esporofitos, los que luego depositan las esporas sobre las fecas de este ganso en las turberas esfagnosas de isla Navarino, donde pueden germinar (Jofré 2009)2. Al mismo tiempo, C. picta constituye la especie de ave más abundante en los ambientes húmedos de en la Reserva de Biosfera Cabo de Hornos (Ibarra et al. 2009a), atributo que la hace formar parte fundamental del grupo de aves que generan un polo de atracción en una incipiente actividad turística de "birdwatching" en el área (Rozzi et al. 2006).

Por otra parte, no fue posible identificar un atributo del hábitat que se relacionara con el éxito o fracaso de los nidos estudiados. Las variables de hábitat no siempre han podido relacionarse con el

\footnotetext{
2Jofré, J. 2009. Fenología del musgo Tayloria dubyi en las turberas de la Reserva de Biosfera Cabo de Hornos: ¿Un Caso de Entomofilia? Tesis Magíster (MSc), Manejo y Conservación de Recursos Naturales en Ambientes Subantárticos, Facultad de Ciencias, Universidad de Magallanes. 111 pp.
} 
éxito reproductivo de una especie, hecho que también ha sido reportado en otros gansos silvestres (Tremblay et al. 1997). En el presente estudio, este hecho sugiere que podrían existir otras variables de hábitat no estudiadas o factores climáticos, que se relacionen significativamente con el éxito o fracaso de los nidos. Sin embargo, también podría estar ocurriendo que C. picta esté presentando un bajo éxito reproductivo por factores extrínsecos al sistema natural, como se señaló anteriormente (e.g., especies invasoras).

Se ha sugerido que el éxito reproductivo en gansos podría ser mayor en zonas altas alejadas de la costa que en ambientes cercanos a ésta (e.g., Ely \& Raveling 1984). En este sentido, este estudio fue desarrollado sólo en zonas costeras de isla Navarino. Algunos de los depredadores que se mencionaron anteriormente son más abundantes en este tipo de ambientes. Por ejemplo, $N$. vison preferiría y sería más abundante en los ambientes costeros por sobre los cuerpos de agua del interior de la isla (Schüttler et al. 2010). Lo mismo ocurre con las aves depredadoras asociadas a ambientes húmedos, las que disminuyen en riqueza y abundancia al alejarse de la costa y siguiendo las cuencas de la isla Navarino (Ibarra et al. 2009a). En este sentido, es relevante poner a prueba esta hipótesis acerca del éxito reproductivo de C. picta en ambientes del interior de la isla, donde los nidos podrían tener una menor presión de depredación, ya que numerosas parejas reproductivas de $C$. picta se dispersan, durante el verano austral, hacia el interior de la isla para su reproducción (Ibarra et al. 2009a).

A partir de este estudio, sugerimos investigar el efecto de los depredadores exóticos sobre el éxito reproductivo de $C$. picta. También, consideramos relevante estudiar la sobrevivencia de volantones, conductas antidepredatorias, decisiones de escape de hembras durante la incubación y el efecto del cambio climático sobre su biología reproductiva. Este último factor estaría afectando significativamente el éxito reproductivo de varias especies de gansos en latitudes altas (Dickey et al. 2008). Programas de educación ambiental pueden contribuir en la promoción de actitudes y prácticas sustentables de cosecha de huevos y en la valoración de las aves de la región. Todos estos elementos podrían ser claves en el reconocimiento de los factores que ponen límites a la población de C. picta en el extremo sur americano. Además, permitirían tener una aproximación más integral en los planes de manejo y conservación de la especie en la $\mathrm{RBCH}$.

TABLA 1. Estudios de biología reproductiva de Chloephaga picta en el extremo sur de Sudamérica y sus respectivos registros de tamaño de puesta, rango de valores, $\mathrm{N}^{\circ}$ de nidos monitoreados y zona de estudio.

\begin{tabular}{ccccccc}
\hline $\begin{array}{c}\text { Tamaño de } \\
\text { puesta }\end{array}$ & Min. & Máx. & Rango & $\begin{array}{c}N^{\circ} \text { Nidos } \\
\text { monitoreados }\end{array}$ & $\begin{array}{c}\text { Zona } \\
\text { estudiada }\end{array}$ & Referencia \\
\hline $6,1 \pm 1,2$ & 4,9 & 7,3 & 3 a 8 & 39 & Isla Navarino & $\begin{array}{c}\text { Ibarra et al. } \\
\text { (este trabajo) }\end{array}$ \\
$5,9 \pm 1,5$ & 4,4 & 7,4 & Desconocido & 126 & Islas Malvinas & Harradine 1977 \\
$6,2 \pm 1,1$ & 5,1 & 7,3 & Desconocido & 20 & Islas Malvinas & Harradine 1977 \\
$4,9 \pm 0,4$ & 4,5 & 5,3 & 3 a 8 & 16 & Islas Malvinas & Quillfeldt et al. 2005 \\
$6,1 \pm 1,1$ & 5 & 7,2 & 3 a 8 & 24 & Islas Malvinas & Summers 1983 \\
\hline 5 & & 7 & 1 a 7 & 41 & Tierra del & Fuego \\
\hline
\end{tabular}




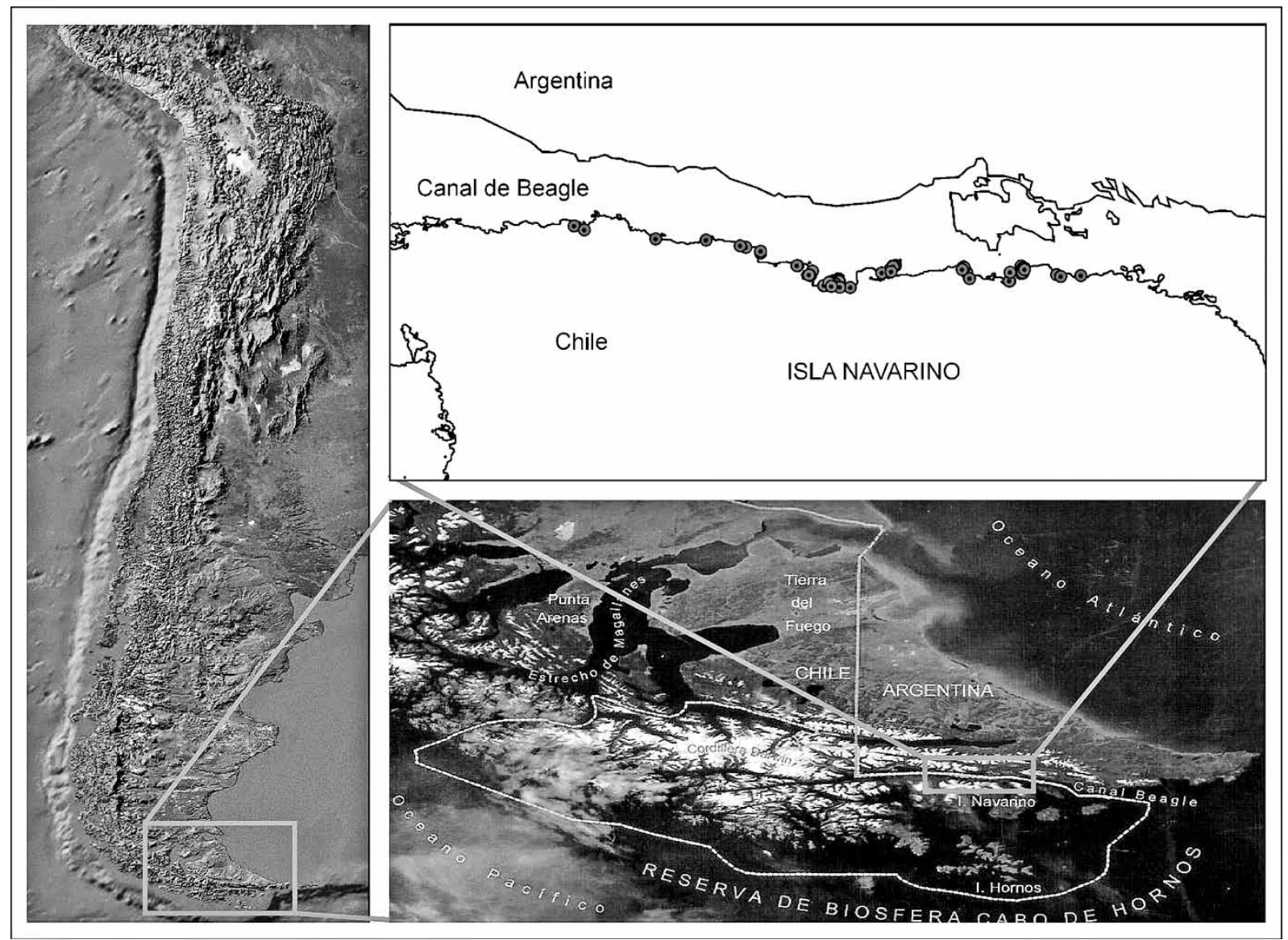

Fig. 1. Mapa del extremo sur de Sudamérica. Se muestra la Reserva de Biosfera Cabo de Hornos, junto con la disposición (puntos en gris) de todos los nidos monitoreados a lo largo de la costa norte de isla Navarino $\left(54-55^{\circ} \mathrm{S}, 67^{\circ} \mathrm{W}\right), \mathrm{Chile}^{\circ}$ 

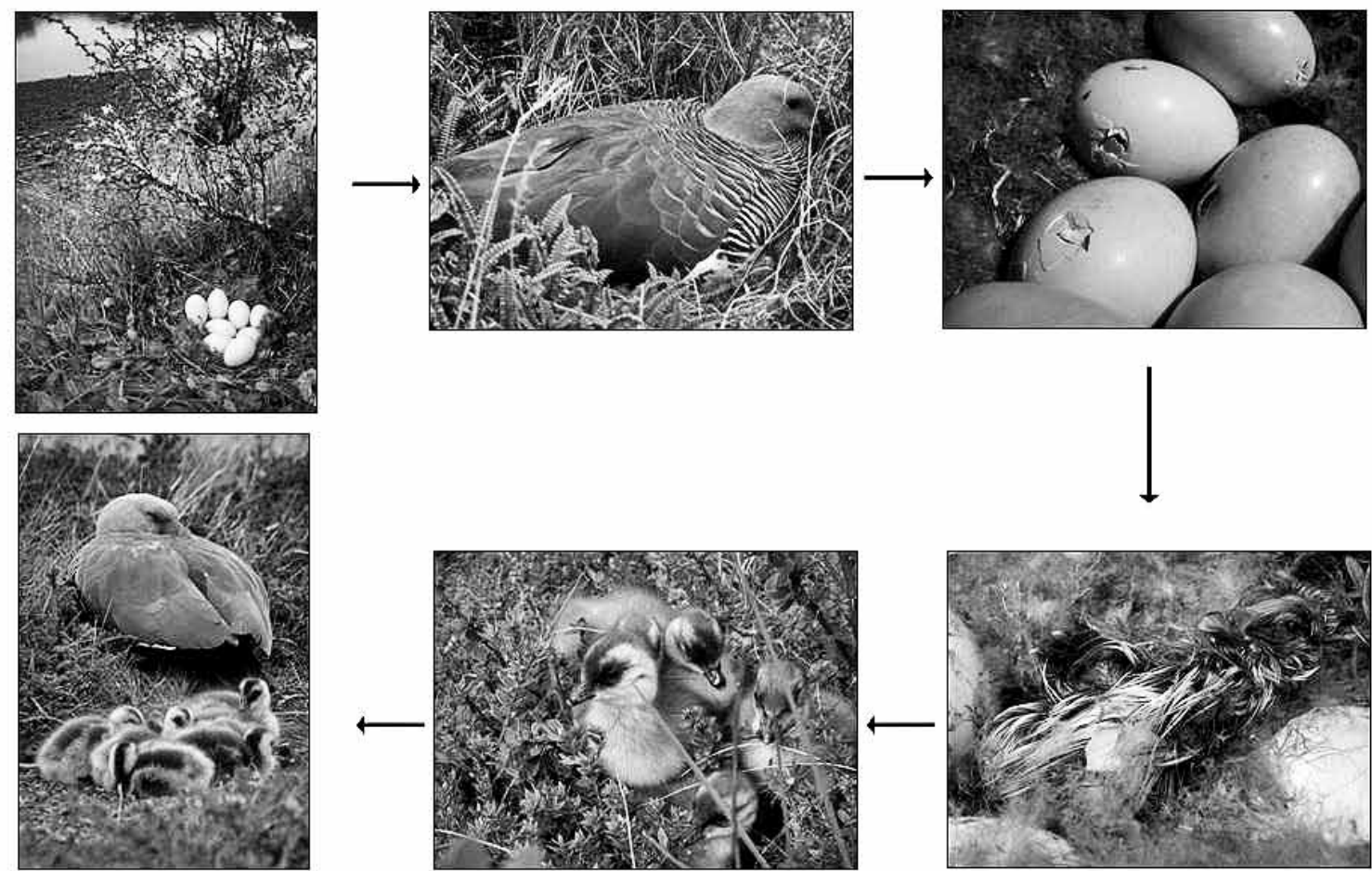

Fig. 2. Ciclo reproductivo de C. picta desde el período de puesta, período de incubación, eclosión y posterior a ésta.

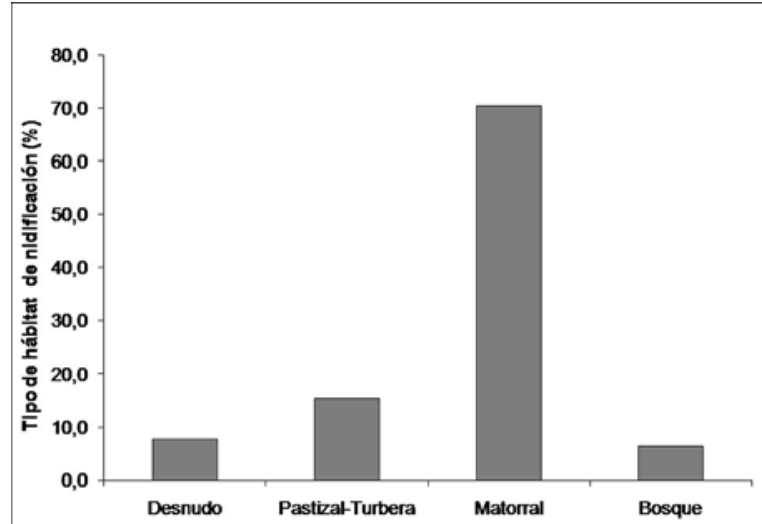

Fig. 3. Tipos de hábitat (\%) dominantes en que nidificó C. picta a lo largo de la costa norte de isla Navarino.

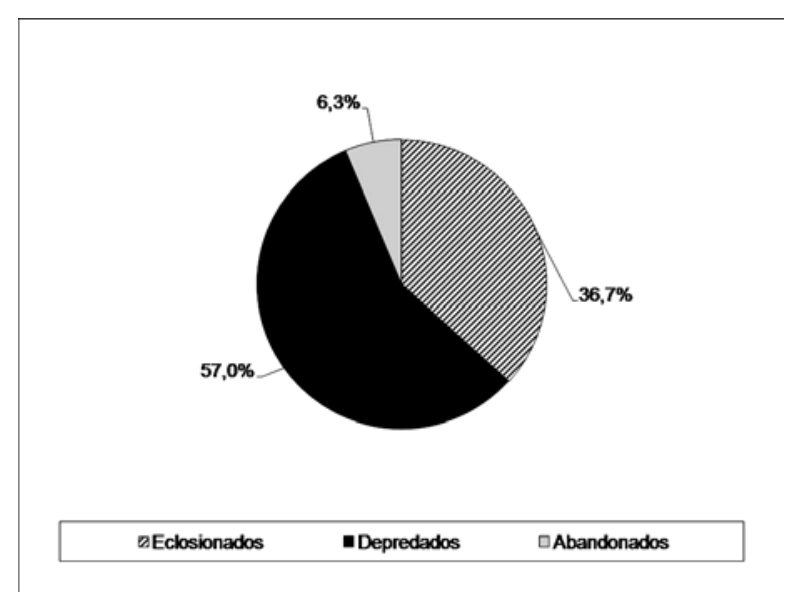

Fig. 4. Estado (\%) en que se encontraron los 39 nidos de C. picta en su última visita de monitoreo, entre los años 2005 y 2007, en isla Navarino. 


\section{AGRADECIMIENTOS}

Al Instituto Milenio de Ecología y Biodiversidad (IEB) (ICM P05-002) y a la Sede Puerto Williams-Universidad de Magallanes, por todo su apoyo logístico-financiero. CONAMA (Región de Magallanes), Wildlife Trust Alliance y el DAAD co-financiaron este proyecto. La investigación fue posible gracias a quienes nos acompañaron en terreno: Tomás Altamirano, Antonia Barreau, Claire Brown, Melisa Gañan, Germán González y José Llaipén. Los autores, junto con Nicolás Galleguillos, tomaron las fotografías del proceso reproductivo. Un especial agradecimiento a Robert Petitpas por su apoyo cartográfico. Agradecemos los comentarios del profesor Claudio Venegas, Dr. Jaime Jiménez y de un revisor anónimo, los que contribuyeron a mejorar versiones tempranas del manuscrito. José Tomás Ibarra es becario CONICYT. Esta investigación forma parte de los programas de conservación e investigación de largo plazo del Parque Etnobotánico Omora en la Reserva de Biosfera Cabo de Hornos, miembro de la Red de Estudios Socio-ecológicos a Largo Plazo de Chile (www.ieb-chile.cl/ltser). Este trabajo está dedicado a la memoria de Paula Ayerdi, perseverante y entusiasta naturalista de las costas de Chile.

\section{LITERATURA CITADA}

Amarasekare, P. 1993. Potential impact of mammalian nest predators on endemic forest birds of western Mauna Kea, Hawaii. Conservation Biology 7: 316-324.

Anderson, C.B., R. Rozzi, J.C. Torres-Mura, S.M. McGehee, M.F. Sherriffs, E. Schüttler \& A.D. Rosemond 2006. Exotic vertebrate fauna in the remote and pristine sub-Antarctic Cape Horn Archipelago region of Chile. Biodiversity and Conservation 10: 3295-3313.

Barros, A. 1971. Aves observadas en las islas Picton, Nueva, Lennox y Navarino oriental. Anales Instituto Patagonia (Chile) 2: 166-180.

Cooper, T.A. 1978. The history and breeding biology of the Canada geese of Marshy Point, Manitoba. Wildlife Monographs 61: 3-87.

Couve, E. \& C. Vidal 2003. Aves de Patagonia, Tierra del Fuego y Península Antártica. Editorial Fantástico Sur Birding Ltda., Punta
Arenas, Chile. 656 pp.

Di Castri, F. \& E. Hajek 1976. Bioclimatología de Chile. Vicerrectoría de Comunicaciones, Pontificia Universidad Católica de Chile. Santiago, Chile. 129 pp.

Dickey, M., G. Gauthier \& M. Cadieux 2008. Climatic effects on the breeding phenology and reproductive success of an arctic nesting goose species. Climate Change Biology 14: 1973-1985.

Earnshaw, E.M. 1973. Entre las aves de Tierra del Fuego. Hornero 11: 203-208.

Ely, C. R. \& D. G. Raveling 1984. Breeding biology of Pacific White-fronted geese. Journal of Wildlife Management 48: 823-837.

Garay, G., W.E. Johnson \& W.L. Franklin 1991. Relative abundance of aquatic birds and their use of wetlands in the Patagonia of southern Chile. Revista Chilena de Historia Natural 64: 127-137.

Godfray, H.C.J., L. Partridge \& P.H. Harvey 1991. Clutch size. Annual Review of Ecology and Systematics 22: 409-429.

Götmark, F. 1992. The effects of investigator disturbance on nesting birds. Current Ornithology 9: 63-104.

Guyn, K.L. \& R.G. Clark. 2000. Nesting effort of northern pintails in Alberta. Condor 102: 619-628.

Harradine, J. 1977. General Ecology. Unpublished report to Ministry of Overseas Development, UK. 130 pp.

Hill, D.A. 1984. Factors affecting nest success in the Mallard and Tufted duck. Ornis Scandinavica 15: 115-122.

Humphrey, P., D. Bridge, P.W. Reynolds \& R.T. Peterson 1970. Birds of Isla Grande (Tierra del Fuego). Smithsonian Institute, Washington D. C, U.S.A.

Ibarra, J.T., R. Rozzi, H. Gilabert, C.B. Anderson, S.M. McGehee \& C. Bonacic 2009a. Dinámica estacional y patrones de distribución de la avifauna asociada a humedales subantárticos en la Reserva de Biosfera Cabo de Hornos (54-55S). Ornitología Neotropical 20: 321-337.

Ibarra, J.T., L. Fasola, D.W. Macdonald, R. Rozzi \& C. Bonacic 2009b. Invasive American mink in wetlands of the Cape Horn Biosphere Re- 
serve, southern Chile: what are they eating? Oryx 43: 87-90.

Jackson S.L., S.H. David \& R.F. Rockwell 1988. The influence of nesting habitat on reproductive success of the Lesser Snow geese. Canadian Journal of Zoology 66: 1699-1703.

Jehle, G., A.A.Y. Adams, J.A. Savidge \& S.K. Skagen 2004. Nest survival estimation: a review of alternatives to the Mayfield estimator. Condor 106: 472-484.

Johnson, D.H. 1979. Estimating nest success: the Mayfield method and an alternative. Auk 96: 651-661.

Jory, J.E., C. Venegas \& W.A. Texera 1974. La avifauna del Parque Nacional "Laguna de los Cisnes" Tierra del Fuego, Chile. Anales Instituto Patagonia (Chile) 5: 131-157.

Klett, A.T., T.L. Shafer \& D.H. Johnson 1988. Duck nest success in the prairie-pothole region. Journal of Wildlife Management 52: 431-440.

Lepage, D., G. Gauthier \& A. Desrochers 1998. Larger clutch size increases fledging success and offspring quality in a precocial species. Journal of Animal Ecology 67: 210-216.

Martínez, D. \& G. González 2004. Las aves de Chile: Nueva guía de campo. Ediciones del Naturalista, Santiago, Chile. 620 pp.

Mayfield, H.F. 1961. Nesting success calculated from exposure. Wilson Bulletin 73: 255-261.

Mayfield, H.F. 1975. Suggestions for calculating nest success. Wilson Bulletin 87: 456-466.

Mickelson, P.G. 1975. Breeding biology of Cackling geese and associated species on the Yukon-Kuskokwim delta, Alaska. Wildlife Monographs 45: 3-35.

Newton, I. 1998. Population Limitation in Birds. Academic Press, London, UK. 596 pp.

Quillfeldt, P., I.J. Strange \& J.E. Masello 2005. Escape decisions of incubating females and sex ratio of juveniles in the Upland Goose Chloephaga picta. Ardea 93: 171-178.

Pisano, E. 1977. Fitogeografía de Fuego-Patagonia Chilena. Comunidades vegetales entre las latitudes $52^{\circ}$ y $56^{\circ} \mathrm{S}$. Anales Instituto Patagonia (Chile) 8: 121-250.

Rozzi, R. \& M. Sherriffs 2003. El visón (Mustela vison Schreber, Carnivora: Mustelidae), un nuevo mamífero exótico para la isla Navarino. Anales Instituto Patagonia (Chile) 31: 97-104.

Rozzi, R., F. Massardo, A. Berghöfer, C.B. An- derson, A. Mansilla, M. Mansilla, J. Plana, U. Berghöfer, P. Araya \& E. Barros 2006. Reserva de Biosfera Cabo de Hornos: Documento de Base para la Incorporación del Territorio Insular del Cabo de Hornos a la Red Mundial de Reservas de Biosfera. Programa MAB-Unesco. Ediciones de la Universidad de Magallanes, Punta Arenas, Chile. 274 pp.

Samelius, G. \& M. Lee 1998. Arctic fox, Alopex lagopus, predation on Lesser Snow geese, Chen caerulescens, and their eggs. Canadian Field-Naturalist 112: 700-701.

Schlatter, R.P., P. Vergara \& M. Briones 2002. El Canquén (Chloephaga poliocephala: Anatidae) en bosques de Tierra del Fuego: distribución y depredadores. Anales Instituto Patagonia (Chile) 30: 61-66.

Schüttler, E., R. Klenke, S. McGehee, R. Rozzi \& K. Jax 2009. Vulnerability of ground-nesting waterbirds to predation by invasive American mink in the Cape Horn Biosphere Reserve, Chile. Biological Conservation. 142:14501460.

Schüttler, E., J.T. Ibarra, B. Gruber, R. Rozzi \& K. Jax 2010. Abundance and habitat preferences of the southernmost population of mink: Implications for managing a recent island invasion. Biodiversity and Conservation 19 (3): 725-743.

Shieck, J.O. \& S.J. Hannon 1993. Clutch predation, cover and the overdispersion of nests of the Willow ptarmigan. Ecology 74: 743-750.

SPSS Inc. 1999. SPSS Base 10.0 for Windows User's Guide. SPSS Inc., Chicago IL.

Summers, R.W. 1983. The life cycle of the Upland goose Chloephaga picta in the Falkland Islands. Ibis 125: 524-544.

Summers, R.W. \& L.G. Underhill 1986. Factors related to breeding production of Brent geese Branta bernicla bernicla and waders (Charadrii) on the Taimyr Peninsula. Bird Study 34: 161-171.

Summers, R.W. \& J. McAdam 1993. The Upland Goose. Bluntisham Books, UK. 162 pp.

Tremblay, J.P., G. Gauthier, D. Lepage \& A. Desrochers 1997. Factors affecting nesting success in greater snow geese: effects of habitat and association with Snowy owls. Wilson Bulletin 109: 449-461.

Wilcove, D.S. 1985. Nest predation in forest tracts and the decline of migratory songbirds. Ecology 66: 1211-1214. 